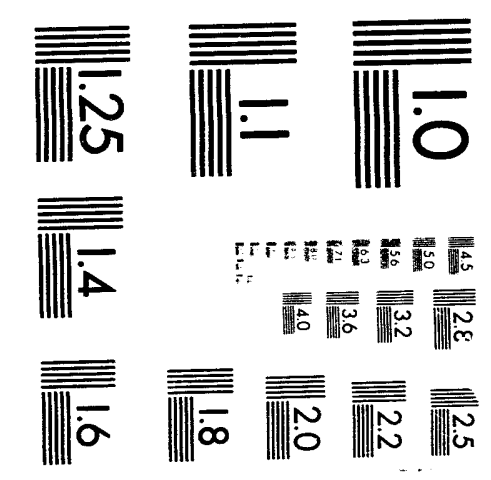



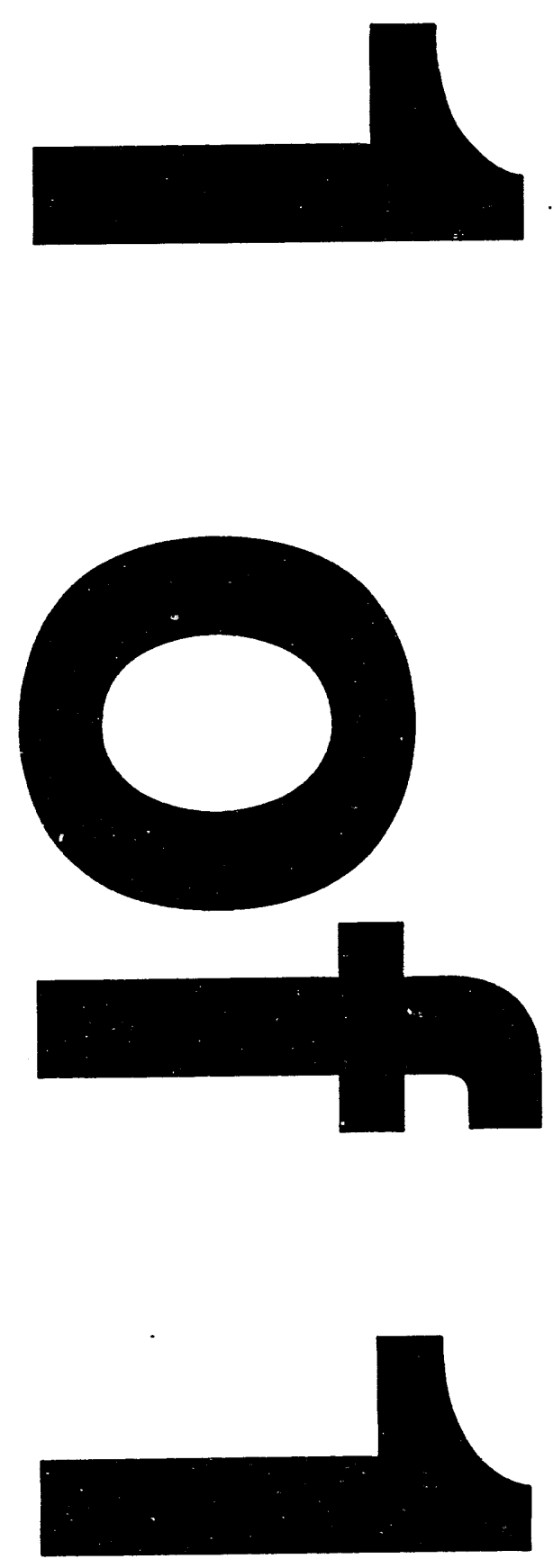
"The subuitted munuecript ho been axtraned oy a contractor of the US. Covernment unce: contract NC. DE-AC05-840R21400. Acoordingly, the US. Goverament retaine a nonexciusive, royalty-free ibone to preblinin or reproduce the pribiinhed form of this contribution, or wiow other to io $\infty$, for U.S. Goveorinent purposes."

\title{
RF Modeling and Design of a Folded Waveguide ICRF Launcher for Alcator C-Mod Tokamak
}

\author{
B y
}

T. S. Bigelow, F. W. Baity, M. D. Carter, P. M. Ryan, J. J. Yugo, C. H. Fogelman, D. J. Hoffman

S. Golovato and P. Bonoli Massachusetts Institute of Technology

Plasma Fusion Center 167 Albany Street

Cambridge, MA 02139

Presented a:

IEEE/NPSS 15th Symposium on Fusion Engineering Hyannis, MA October 11.15, 1993

Prepared for the

Fusion Energy Division

Prepared by

Oak Ridge Nationai Laboratory

Post Office Box 2008

Oak Ridge, TN 37831-6285 USA

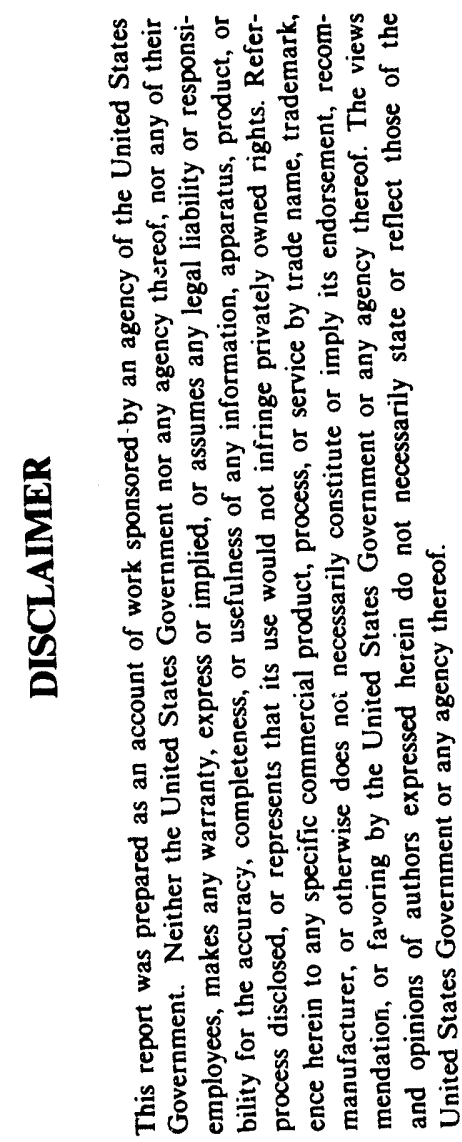
Managed by:

MARTIN MARIETTA ENERGY SYSTEMS, INC. for the

U.S. DEPARTMENT OF ENERGY

under contract DE-AC05-84OR21400 
The subrimed menuecript has bean

\title{
RF MODELING AND DESIGN OF A FOLDED WAVEGUIDE LAUNCHER FOR THE ALCATOR C-MOD TOKAMAK
}

\author{
T. S. Bigelow, C. F. Fogelman, F.W. Baity, M. D. Carter, D. J. Hoffman, P.M. Ryan, \\ J.J. Yugo, Oak Ridge National Laboratory, P.O. Box 2009, Oak Ridge, TN 37831-8071 \\ S. N. Golovato, P. Bonoli, Massachusetts Institute of Technology, Cambridge, Ma. 02139
}

\section{ABSTRACT}

The folded waveguide (FWG) launcher is being investigated as an improved antenna configuration for plasma heating in the ion cyclotron range of frequencies (ICRF). A development FWG launcher was successfully tested at Oak Ridge National Laboratory (ORNL) with a low-density plasma load and found to have significantly greater power density capability than current strap-type antennas operating in similar plasmas. To further test the concept on a high density tokamak plasma, a collaboration has been set up between ORNL and Massachusetts Institute of Technology (MIT) to develop and test an 80-MHz, 2-MW FWG on the Alcator C-Mod tokamak at MIT. The radio frequency (rf) electromagnetic modeling techniques and laboratory measurements used in the design of this antenna are described in this paper. A companion paper [1] describes the mechanical design of the FWG.

\section{INTRODUCTION}

The standard FWG launcher [2] is a resonant cavity formed with a half wavelength section of thin rectangular waveguide folded in one dimension and completely enclosed on the back end as shown in Fig. 1. Folding allows a large, low frequency waveguide to fit into the available port dimensions on tokamak devices. The folding process is implemented by stacking flat vanes with rounded tips on alternate sides of a rectangular box. On the plasma facing end, the FWG is partially shorted with a polarizing plate that has openings between alternate vanes to allow in-phase polarized magnetic fields to couple to the plasma. The magnetic fields penetrate into the plasma edge and couple to plasma fast waves when the waveguide is oriented with the rf magnetic fields in the toroidal direction. There is a small ripple component in the magnetic field pattern caused by the spaces between polarizing plate openings. This ripple is not expected to adversely affect the plasma coupling since its amplitude dies out quickly away from the FWG. Antenna patterns for the FWG resemble those of current strap antennas outside the ripple region.. Transverse electromagnetic fields inside the FWG vary

Research sponsored by the Office of Fusion Energy, U.S. Department of Energy, under contract DE-AC05-840R21400 with Martin Marietta Energy Systems sinusoidally along its length. The electric field is maximum at the center, zero at the back wall, and very low at the plasma end. The magnetic field is maximum at the back wall and polarizing plate. The low electric field and high magnetic field at the polarizing plate is optimum for plasma coupling.

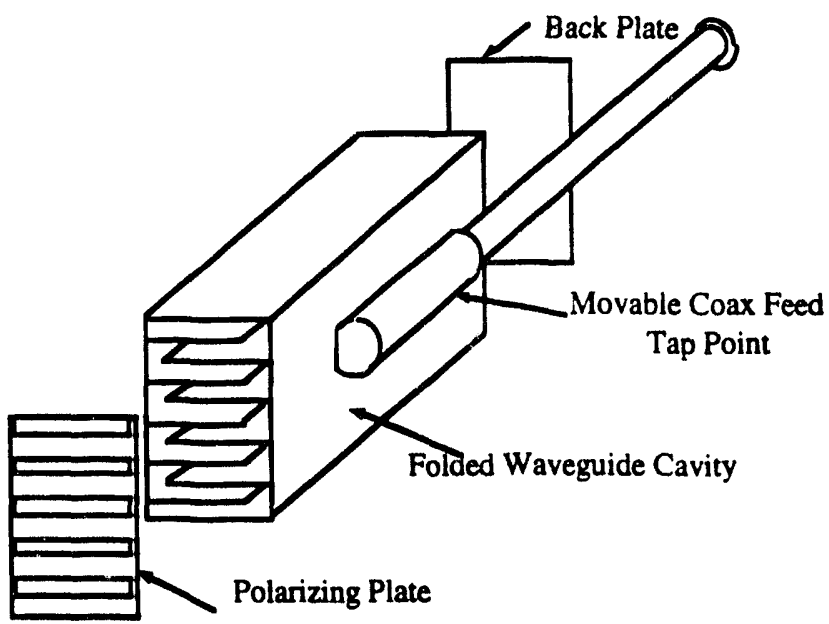

\section{Fig. 1 Folded wayeguide launcher configuration}

The FWG launcher is under devslopment as an ICRF antenna because of several features trat may be particularly advantageous in a tusion reactor environment: (1) tests of a development FWG at ORNL have d/amonstrated a factor of $\sim 5$ increase in power density $C^{-3 p a b}{ }^{\prime \prime}$ oy for essentially identical plasma loads [3]; ( 2 ;' 'snce the $\mathrm{F}$ WG has no center conductor, ceramic insulatir's supports aje not raquired close to the plasma; (3) the j does ncı $n$ qu' a Faraday shield and is inherently robus: $1 \mathrm{~g}$, ist plasma disruption induced forces; (4) the input snpedance can he internally matched eliminating the need for external tunir ? vipment; (5) there is very little mutual coupling betweer a cent FWG apertures which simplifies phasing urray", and (6) the FWG can be designed to provide radial position acisjastment capatility.

The main disadvantage of the standard FWG launcher is its narrow bandwidth. It is necessary to vary the cavity length or width to change resonant frequency. This requires that additional mechanical hardware be incorporated inside the FWG which is not desirable in a reactor antenna. Reliability of frequency tuning and matching hardware mounted inside a FWG needs to be established. For some reactor scenarios, narrow band operation may be acceptable. Other configurations of the FWG have been devised such as the quarter-wavelength FWG cavity version, a folded stripline, and 
a stacked stripline [4] may offer the potential for wider bandwidth operation using either external matching or less complex internal tuning hardware.

Although the FWG has many potential advantages, it has not yet been tested with a high-density tokamak plasma. Theretore, a collaborative development and testing program has been established between ORNL and MIT to test a FWG on the Alcator C-Mod tokamak. An 80-MHz, 2-MW FWG launcher is currently being designed at ORNL and is scheduled to be tested on C-Mod beginning in late 1995. The C-Mod FWG will have a movable feed point for impedance matching and will have radial position adjustment capability. A vacuum tank will enclose the FWG and if power will be coupled through a 9 -in., $50-\Omega$ vacuum teedthrough. Design of the FWG is being based on techniques derived from previous FWG design projects and an $80-\mathrm{MHz}$ development FWG that was tested to $1 \mathrm{MW}$. Objectives of the C-Mod FWG testing program will be to measure the plasma loading, power density, plasma impurity generation, reliability, and heating capabilities of the folded waveguide with a high density divertor-type plasma.

\section{FWG RF DESIGN PROCEDURE}

The parameters required for the design and analysis of the FWG cavity are the resonant frequency, the electric and magnetic fields in the cavity, the rf losses (or cavity Q), and ultimately the fields around the polarizing plate openings and the feed structure. From the cavity field values, estimates can be generated for the plasma loading, the power launch spectrum, and the power handling capability.

The FWG launcher is a complicated three-dimensional device. which makes modeling difficult especially when the plasma-antenna interaction is being considered. Fortunately. the behavior of the FWG cavity is only slightly perturbed by the plasma load, which allows the FWG cavity to be designed independent of plasma conditions by using modified unfolded waveguide cavity models or commercially available numerical codes. More accurate calculation of the cavity resonant frequency and electric fields and currents around the FWG internal vane tips is best handled by using a 2-D modeling technique for an infinite length waveguide such as the finite element or the finite-difference time-domain method (FDTD). Nonuniform grids with higher point density near the vane tips are desirable for greater accuracy. Since the numerical techniques can require hours of computing time for each case, a simpler approximate analytical technique is useful for the initial FWG design and has been implemented in a spreadsheet. Accurate modeling of the plasma loading, launch spectrum, and currents on the antenna front facing the plasma requires more complicated plasma-antenna interaction codes that are under development at ORNL [5] and elsewhere.

On the Alcator C-Mod tokamak. a transmitter operating irequency of $80 \mathrm{MHz}$ and port dimensions of $203 \mathrm{~mm}$ horizontal by $438 \mathrm{~mm}$ vertical are available. The waveguide cutoff frequency is set by the effective width of the folded iavity and must be sutficiently below the operating frequency to allow a reasonably short waveguide box. If too many vanes are required, the vane-to-vane spacing will provide insufficient clearance for the input power feed "vane toucher." Uneven vane spacing can be used to allow more room for the fieed at the central vane. For the C-Mod case, 13 evenly spaced vanes of $6.35 \mathrm{~mm}$ thickness and $12.7-\mathrm{mm}$ walls have heen chosen, which produces a cutotf frequency of $69 \mathrm{MHz}$. This size allows $6 \mathrm{~mm}$ clearance between the outside of the FWG box and the C-Mod port sidewalls.

The cutoff frequency of the FWG is set by the effective width of the cavity, which to first order, is simply the centerline length of the meandering waveguide path. The sharp $180^{\circ}$ hairpin turns at each vane tip combined with the square corners on the opposite walls act as capacitive discontinuities in the line that shorten its effective length. A simple but fairly accurate analytical model of the effective length has been developed based on an electrostatic approximation of the fields near a rounded vane tip, which is elevated in potential above the nearby rectangular corner. The half-potential surtace is pulled closer to the vane. effectively shortening the path length over the original centerline as shown in Fig 2. A spreadsheet program to calculate the effective waveguide width has been implemented, which allows quick estimates of the FWG resonant frequency. This spreadsheet predicts a resonant frequency of $386.35 \mathrm{MHz}$ for a 9- vane scale model test FWG, which is very close to the measured value of $386.37 \mathrm{MHz}$.

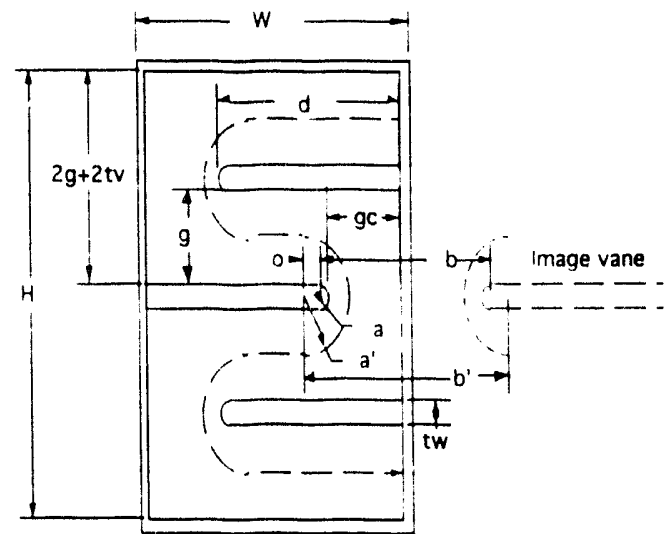

Fig. 2 GEOMETRY USED IN FWG DESIGN CALCULATOR

\section{FWG ANALYSIS TECHNIQUES}

Several analysis tools have been applied to the FWG without a plasma load. Two commercially available numerical codes, EMAS [6] and VARGUS [7], have been used to solve for the eigenmodes of a basic FWG cavity that is closed on both ends and has square-ended vanes. Both codes have successfully converged for several of the lowest order modes of scale model FWG's. The resonant frequencies agree closely with values measured on a test unit (which actually has rounded vane tips).

The VARGUS code uses a irequency-domain eigenmode solver for the finite difference form of Maxwell's equations. A 
$77 \times 26 \times 21(42,042$ point $)$ grid was used to model the test FWG, and a resonant frequency of $386.2 \mathrm{MHz}$ was predicted. which agrees well with the measured resonant frequency of $386.37 \mathrm{MHz}$. This code and grid configuration will also be used to model the C-Mod FWG unit. A surface plot of VARGUS output for the electric field component parallel to the vanes in a cross section slightly above the vanes and on the midplane of the $386-\mathrm{MHz}$ model is shown in Fig. 3. The electric field is peaked near the vane tips and maximum along its length at the center point of the FWG. The electric fields near the corners of the square vane tips appear as spikes in the output data.

The EMAS code uses finite elements to solve the vector potential form of Maxwell's equations. A grid that is tailored to match the geometry of the problem can be set up to improve the accuracy. A simple FWG box case has been successfully solved with this code. This code has a large number of features and advanced graphical output plotting capabilities.

\section{FWG-PLASMA INTERACTION}

The VARGUS and EMAS numerical electromagnetic codes are capable of analyzing the FWG box, and both codes can probably be extended to include the open polarizing plate by adding an additional box around the face of the antenna. Plasma loading can be simulated by adding absorbing material or absorbing boundary conditions near the polarizing plate. The modeling of a realistic tokamak plasma is very difficult with this type of code due to the complicated nature of the rapidly varying plasma dielectric and the large relative size of the plasma. Although the plasma and FWG internal structure are almost decoupled, the small coupling that does exist represents the antenna loading. Ultimately, a model that includes both FWG and plasma regions with high accuracy is desirable.

The MANTIS code developed at ORNL by Carter [5] for analyzing the interaction between current strap antennas and plasma has been adapted to the FWG case. This code uses a slab plasma model with toroidal and poloidal periodicity to estimate the plasma surface impedance for a realistic density profile and edge plasma. A grid of current elements is set up to approximate the current distribution flowing on the polarizing plate and back into the FWG on each vane surface. The power coupled to the plasma and the launch spectrum is calculated by enforcing continuity of the fields at the plasma edge in Fourier space. The power coupled to the plasma vs. if magnetic field at the polarizing plate for the C-Mod FWG is estimated to be $140 \times 10^{9} \mathrm{~W} / \mathrm{Tesla}^{2}$ using the modified MANTIS code. A relationship between the polarizing plate magnetic field and internal electric fields can be estimated using the MANTIS code and also the numerical codes. Laboratory measurements of the FWG fields can be used to check these results [8].

A FWG code based on waveguide modal analysis using unfolded waveguide modes was written by Owens [2]. This code models the plasma region in front of the polarizing plate as a lossy dielectric and is quite useful in studying the field distribution inside and outside the FWG cavity. Further accuracy may be possible by using this code or the MANTIS code with numerical solutions for the actual modal fields inside the FWG calculated by the EMAS or VARGUS codes.

\section{CONCLUSION}

The rf design and analysis techniques for FWG launchers that are being applied to develon a FWG for the C-Mod tokamak have been quite successful in the preliminary design stage. Numerical techniques have yielded accurate results for the FWG cavity fields. Improved simulations using finer grids and a polarizing plate are expected to be performed soon. A plasma-FWG interaction model is being developed and will be used for predicting loading and launch spectrum. Detailed measurements of resonant frequency and magnetic field patterns for a $386-\mathrm{MHz}$ test unit have been performed and are available for code verification.

\section{REFERENCES}

[1] C. H. Fogelman et al., paper this proceedings.

[2] T. L. Owens, IEEE Trans. Plasma Sci., Vol. PS-14 (6), pp. 934-46, 1986.

[3] F.W. Baity et al., "Radio-Frequency Power in Plasmas," AIP Conf. Proc. 244, pp. 298-301, 1991.

[4] W. L. Gardner et al., "Radio-Frequency Power in Plasmas," AIP Conf. Proc., 1993 (unpublished)

[5] M.D. Carter et al., "Multiple Antenna Implementation System," ORNL/TM-12264, 1993.

[6] EMAS - a proprietary product available from the McNeilSchwindler Corp., Milwaukee, Wisconsin.

[7] VARGUS - a proprietary product available from SAIC, McLean, Virginia.

[8] G. R. Haste, et al., "The folded waveguide - a high frequency rf launcher," Fus. Engr. \& Des. (unpublished). 
NINE VANE FOLDED WAVEGUIDE (5:1 Scale)

First Eigenmode at $386.2 \mathrm{MHz}$

Dimensions: $60.7 \times 13.5 \times 5.5 \mathrm{~cm}$ Grid: $23 \times 79 \times 28$

Poloidal Field Component $E_{y}$ at Toroidal Location $z=1.12 \mathrm{~cm}$ (vane tips)

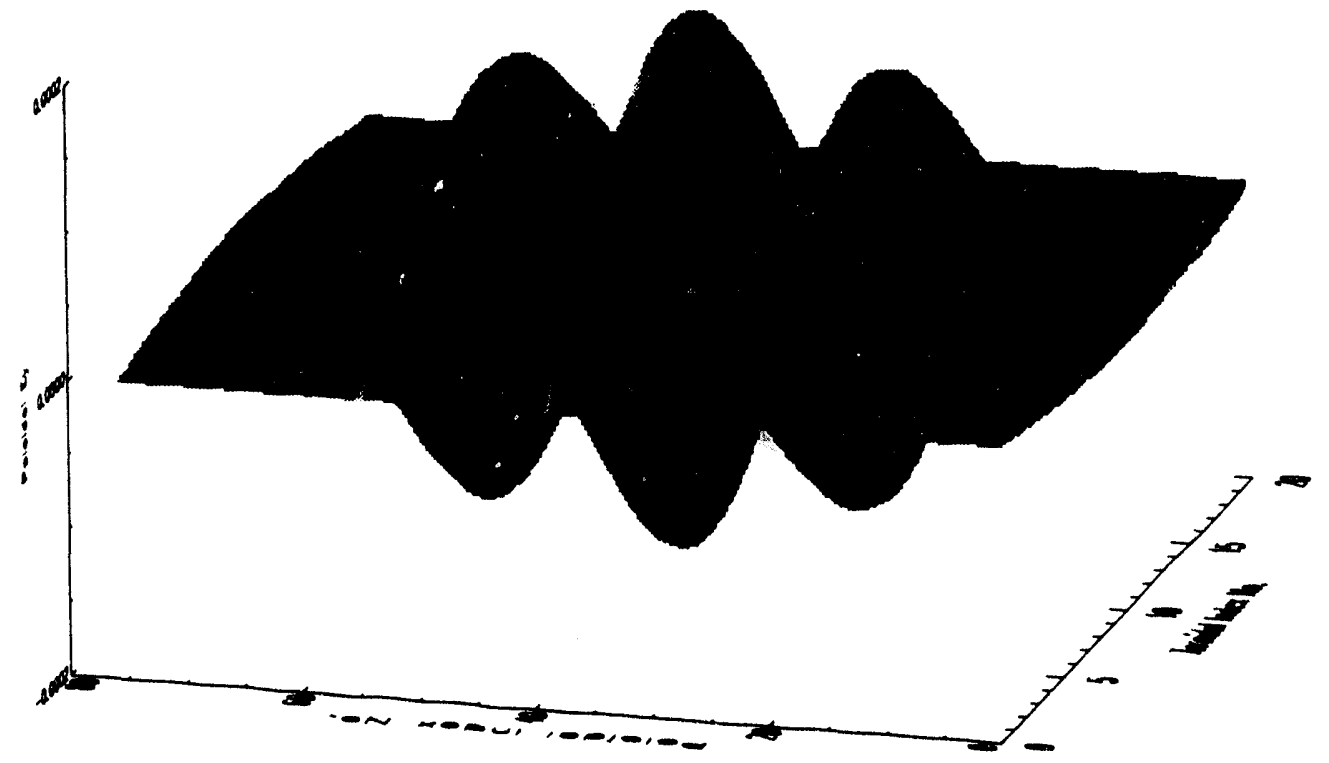

Poloidal Field Component $E_{y}$ at Toroidal Location $z=2.7 \mathrm{~cm}$ (midplane)

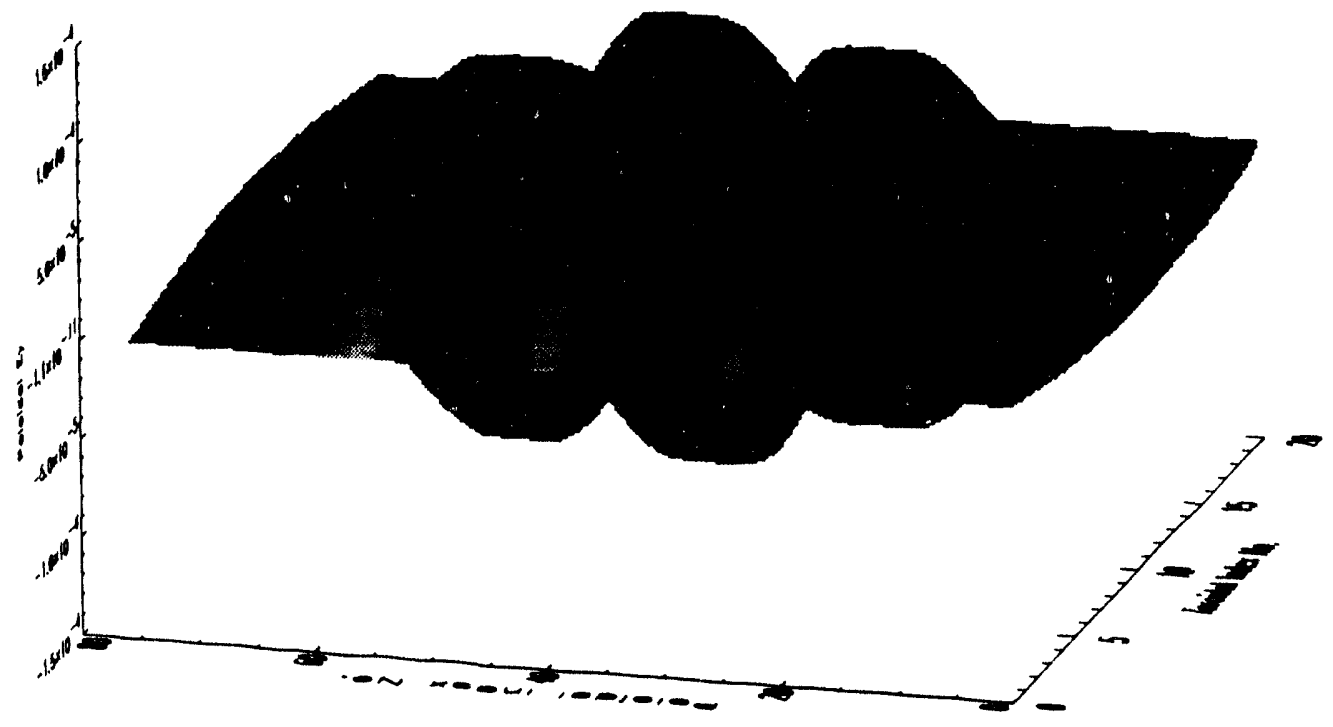

Fig. 3 Calculations of the poloidal electric field component, $\mathrm{E}_{\mathrm{y}}$ for the $386 \mathrm{MHz}$ FWG using VARGUS 

